\title{
Psychiatry in the Foundation Programme: an overview for supervisors ${ }^{\dagger}$
}

\author{
Jennifer Perry, Howard Ryland, Lesley Thoms \& Ann Boyle
}

\begin{abstract}
SUMMARY
Health Education England's 2014 report Broadening the Foundation Programme has led to a rapid increase in the number of psychiatry placements for foundation doctors. This will have implications for existing psychiatrists in that they will start to teach, train and supervise foundation doctors. This article outlines how to develop high-quality foundation posts and how to support doctors in meeting the foundation curriculum competencies, which include experience of working in multidisciplinary teams and developing communication skills. The knowledge and skills gained in psychiatry placements will be valuable to all doctors, no matter what their future career intentions.
\end{abstract}

\section{LEARNING OBJECTIVES}

- Describe the recent changes to the Foundation Programme in psychiatry and how they will affect supervisors

- Learn how high-quality placements for foundation doctors can be developed

- Recognise how foundation doctors can be supported to achieve the generic and mental health-specific competencies of the foundation curriculum in psychiatry placements

\section{DECLARATION OF INTEREST}

None

This article gives a succinct, practical and accessible overview of the changes to the Foundation Programme. Much of the information is based on the Royal College of Psychiatrist's A Guide to Psychiatry in the Foundation Programme for Supervisors (Boyle 2015) and a BJPsych Bulletin paper entitled 'The expansion of the Foundation Programme in psychiatry', which describes the changes and focuses on the support being put in place by the College to ensure that foundation doctors have a good experience of psychiatry (Perry 2016).

\section{Introduction to the Foundation Programme}

The Foundation Programme (Box 1) was instituted in 2005 and brought together the pre-registration house officer grade and the first year of the senior house officer grade (UK Foundation Programme Office 2015). This created an integrated 2-year programme, governed by a single curriculum produced by the Academy of Medical Royal Colleges (2016). The majority of students completing undergraduate medical training in the UK will enter the Foundation Programme directly after leaving medical school.

The Foundation Programme is coordinated nationally by the UK Foundation Programme Office, which is commissioned by the four UK national health departments: Health Education England (HEE), NHS Education for Scotland (NES), the Northern Ireland Medical and Dental Training Agency (NIMDTA) and the Wales Deanery. These four bodies are responsible for ensuring that the Foundation Programme is delivered across the UK in accordance with the standards set by the General Medical Council (GMC). Foundation schools are the structures through which each of the education authorities (HEE/NES/NIMDTA/Wales Deanery) delivers foundation training. Training is provided by local education providers (LEPs) through primary, secondary and academic placements (UK Foundation Programme Office 2016). Outside of the UK, the Foundation Programme has been

BOX 1 The aims of the Foundation Programme

- Build on undergraduate education by instilling recently graduated doctors with the attributes of professionalism and the primacy of patient welfare

- Provide generic training that ensures that foundation doctors develop and demonstrate a range of essential interpersonal and clinical skills for managing both acute and long-term conditions

- Provide the opportunity to develop leadership, team working and supervisory skills

- Provide each foundation doctor with a variety of workplace experience in order to inform their career choice (UK Foundation Programme Office 2016)

ARTICLE

Jennifer Perry is an ST5 in general adult psychiatry with the South London and Maudsley NHS Foundation Trust. Howard Ryland is an ST5 in forensic psychiatry with the West London Mental Health NHS Trust. Lesley Thoms is a Clinical Fellow in Leadership and Management at the Royal College of Psychiatrists. Ann Boyle is a consultant old age psychiatrist with Leicestershire Partnership NHS Trust, Associate Postgraduate Dean of Health Education England East Midlands, and a Specialist Advisor for the Foundation Programme, Royal College of Psychiatrists. Correspondence Dr Jennifer Perry, c/o BJPsych Advances, The Royal College of Psychiatrists, 21 Prescot Street, London E1 8BB, UK. Email: jenperry@doctors.org.uk

\section{Copyright and usage}

(C) The Royal College of Psychiatrists 2017.

${ }^{\dagger}$ For a related article see pp. 131-142, this issue. 
replicated in Malta. The Maltese programme was launched in 2009 and it uses the same curriculum and provides the same educational and training opportunities (Foundation Programme Malta 2016). As far as we are aware, the Foundation Programme is not being used elsewhere. However, in other countries, such as Australia and the USA, graduates undertake an internship during which they are supervised until they gain full registration (Wilson 2015).

Foundation training consists of a series of placements over 2 years in a wide variety of specialty areas, including psychiatry. Each placement lasts a minimum of 4 months and a maximum of 6 months. Foundation doctors complete a 'Preparing for Professional Practice Programme' or shadowing period before starting their placements (Miles 2015).

\section{Recent changes to the Foundation Programme}

A review of the Foundation Programme in 2010 by Professor John Collins recommended that the placements offered should represent a wider range of specialties (Collins 2010). This recommendation helped inform a programme of work called 'Better Training, Better Care', under the auspices of

BOX 2 Recommendations of the Broadening the Foundation Programme report

\section{Recommendation 1}

'Educational supervisors should be assigned to foundation doctors for at least one year, so they can provide supervision for the whole of Foundation Stage 1 (F1) Foundation Stage 2 (F2), or both years.'

Recommendation 2

'Foundation doctors should not rotate through a placement in the same specialty or specialty grouping more than once, unless this is required to enable them to meet the outcomes set out in the Curriculum. Any placements repeated in F2 must include opportunities to learn outside the traditional hospital setting.'

\section{Clarification}

Where the experience will be significantly different between the posts, then two posts within a specialty grouping are permitted - for example, acute internal medicine (admitting) posts and general medicine (ward-based) posts. In such circumstances, the subspecialties should not be the same.

\section{Recommendation 3}

'a At least 80 per cent of foundation doctors should undertake a community placement or an integrated placement from August 2015.

b All foundation doctors should undertake a community placement or an integrated placement from August 2017. It should be noted that both community and integrated placements are based in a community setting, and that an acute-based communityfacing placement is not a substitute.'

Clarification

Psychiatry posts in mental health hospitals that are not on the site of an acute hospital will be counted as being community posts. Psychiatry posts located within acute hospitals where the trainee will have the opportunity to look after patients with long-term psychiatric conditions and/or work with community services/MDTs will also be counted as community posts. Liaison psychiatry would fall under this definition

(Health Education England 2014: p. 9)
Medical Education England and subsequently HEE (Health Education England 2015). In February 2014, HEE released its report entitled Broadening the Foundation Programme (Health Education England 2014). This set out radical changes to the Foundation Programme (Box 2), constituting a move away from the traditional 6 months of medicine and 6 months of surgery that used to make up the typical pre-registration house officer year (Bleakley 2002).

The HEE report recommended that, by 2017, $100 \%$ of doctors should rotate through a community placement in their Foundation Programme (all psychiatry placements are counted as community placements). The target set by the psychiatry task force is that $45 \%$ of all doctors completing the programme in England, with $22.5 \%$ in each of the 2 years, should undertake a placement in psychiatry.

The report specifically focused on the need to achieve parity of mental and physical health. Mental illness is responsible for $23 \%$ of the burden of disease in England, affecting one in four people and costing around $£ 105$ billion each year (Davies 2014). There is a need for this parity to be reflected in the training of doctors. In 2012, figures showed that psychiatry represented $6 \%$ of core training posts, and only $2 \%$ of foundation year 1 (FY1 or F1) posts. This contrasted sharply with the situation in surgery, which in the same year had 9\% of core training posts and 37\% of FY1 posts (Health Education England 2014).

The report also noted that, despite initial negative attitudes towards placements in psychiatry among many foundation doctors, these were mostly overturned by the end of the placements. Participants identified many positive aspects, including opportunities to learn about wholeperson care, high-quality clinical supervision and the chance to develop skills in history-taking and mental state examination.

\section{What does this mean for psychiatrists?}

The principal implication of these changes for the medical workforce in psychiatry is a large and rapid increase in the number of foundation placements in psychiatry. Many more consultants will become supervisors of foundation doctors. Other non-consultant grade psychiatrists will encounter foundation doctors in their teams and many will also be involved in their training. Many psychiatrists willnot havehad experience of working with foundation doctors before. Psychiatrists are used to providing core and specialist training; with foundation doctors they will need to provide more generalist training which meets the requirements 
of the Foundation Programme curriculum. A key aim of the Foundation Programme in psychiatry is that doctors will be able to develop generic, transferrable skills that will be useful in their future careers, regardless of the specialty.

\section{What do psychiatrists need to know about foundation doctors?}

What is the difference between foundation year 1 (FY1) and foundation year 2 (FY2)?

Foundation year 1 enables medical graduates to begin to take supervised responsibility for patient care and consolidate the skills they have learned at medical school. Satisfactory completion of FY1 allows the relevant university (or their designated representative in a foundation school) to recommend to the GMC that the foundation doctor can be granted full registration (GMC 2015). It is worth noting that this may change in the future, as the 'Shape of Training' review led by Professor David Greenaway (Shape of Training 2013) has recommended that full registration should move to the point of graduation from medical school.

When they first start, most FY1 doctors will be very inexperienced and will require a substantial amount of support from their supervisors and the wider multidisciplinary team (MDT). This will change as the year goes on and they move into FY2, as they will start to develop skills, knowledge and confidence in their clinical work. FY2 doctors remain under clinical supervision, but take on increasing responsibility for patient care. They begin to make management decisions, develop their core generic skills and contribute more to the education/training of the wider healthcare workforce. At the end of FY2, doctors should have started to demonstrate clinical effectiveness, leadership and decision-making responsibilities (Goodyear 2014). Satisfactory completion of FY2 will lead to the award of a Foundation Programme Certificate of Completion (FPCC), which indicates that the foundation doctor is ready to enter a core, specialty or general practice training programme (UK Foundation Programme Office 2016).

\section{What is the difference between foundation training and psychiatric specialty training?}

It is important to remember that foundation doctors are not learning to be psychiatrists, unlike core and specialty psychiatry trainees. The aim of the programme is to give foundation doctors a meaningful experience of psychiatry and to allow them to achieve the Foundation Programme competencies. However, if foundation doctors have an interest in psychiatry, they can be supported in accessing additional learning opportunities.
All trainees play a key role in the delivery of National Health Service (NHS) care and it is important that foundation doctors 'learn by doing', as they will learn more effectively when they are responsible for their actions (Swanwick 2014). FY1 doctors are new medical graduates and will be inexperienced compared with core psychiatry trainees. Therefore they need to be well supervised to allow them to develop as doctors while ensuring patient safety.

\section{What are the boundaries of responsibility for foundation doctors?}

Although technically FY1 doctors can prescribe anywhere, including in the community, the Royal College of Psychiatrists recommends that those in psychiatry placements should only prescribe in an in-patient setting. However, local exceptions to this may occur if adequate safeguards are in place (Boyle 2015). Although FY1 doctors may work out of hours, they should not be assigned to work on the same rotas as core trainees, and special arrangements should be made to ensure that they are adequately supported and supervised. In practice, this means that there must be a senior colleague on site. This colleague could be a senior nurse, as long as they have the necessary knowledge and skills to advise the foundation doctor appropriately (Boyle 2015).

\section{How can supervisors ensure that their posts are of high quality?}

High-quality training in new and existing foundation posts requires careful planning and execution in order to maintain patient safety, inspire trainees and preserve enthusiasm (Royal College of Psychiatrists 2016). There are many ways in which psychiatrists can develop highquality placements for foundation doctors. Examples are detailed below.

\section{Shadowing and induction}

Before beginning their first placement, FY1 doctors should undertake a shadowing period of at least 4 days' duration (which includes induction) overseen by their clinical supervisor (UK Foundation Programme Office 2016).

All new foundation doctors should normally have sat a Prescribing Safety Assessment (PSA) before commencing the programme. UK medical students should have been given the opportunity to sit the PSA during their degree course. Appointees from non-UK medical schools will be offered the opportunity to sit the PSA before or during their shadowing period where possible. Foundation doctors who have not passed the PSA 
before starting the programme are offered support and are required to sit/re-sit the assessment. All foundation doctors are required to pass the PSA within the 2 years immediately preceding the date on which they are signed off as having completed their FY1 year (UK Foundation Programme Office 2016).

Foundation doctors should have an induction from their foundation school and their employer/ local education provider. To ensure patient safety, all rotations should also initiate foundation doctors through a formal induction to the department and place of work.

Consultants should discuss the level of training and competency of the new incoming doctor with members of the MDT, managing expectations and clarifying the duties and responsibilities of foundation doctors. Team members should be told explicitly what is and what is not expected of a foundation doctor: for example, FY1 doctors should not undertake unsupervised section 136 Mental Health Act assessments and they do not have powers of detention.

Supervisors should organise a workplace induction process and timetable which ensures that the foundation doctor is introduced to all team members and is familiarised with the working environment (and health and safety procedures). The supervisor should give the foundation doctor an overview of key issues in psychiatry such as the Mental Health Act, the Mental Capacity Act, and risk and safeguarding, which can be expanded on in supervision sessions.

The MDT should be involved in the foundation doctor's induction and placement. Members of the team will be able to support the doctor's training, for example through conducting joint assessments with the doctor, undertaking supervised learning events (SLEs) and giving feedback on their progress.

\begin{tabular}{|l|l|l|l|l}
\hline Supervised learning events (SLEs) \\
\hline Directly observed interactions & $\begin{array}{l}\text { - Mini-clinical evaluation exercises (mini-CEXs): a mini-CEX is an } \\
\text { observed clinical encounter, e.g. observing the doctor taking a } \\
\text { patient history } \\
\text { - Direct observation of procedural skills (DOPS): the primary aim of a } \\
\text { DOPS is to give feedback on the doctor's interaction with a patient } \\
\text { while performing a procedure, e.g. an ECG or taking bloods; the } \\
\text { secondary aim is to demonstrate the progression of procedural } \\
\text { skills }\end{array}$ \\
\hline Case-based discussions (CBDs) & $\begin{array}{l}\text { - A CBD is a structured discussion of a clinical case managed by the } \\
\text { doctor; it has a focus on clinical reasoning }\end{array}$ \\
\hline Developing the clinical teacher & $\begin{array}{l}\text { - This tool aims to assess and develop the doctor's teaching and } \\
\text { presentation skills by observing a teaching session }\end{array}$ \\
\hline
\end{tabular}

The components of supervised learning events (SLEs) for foundation doctors in foundation years 1 and 2 (Academy of Medical Royal Colleges 2016).

\section{Teaching}

Supervisors should consider the specific learning needs of foundation doctors and try to align teaching opportunities with their curriculum. All foundation doctors are required to attend their mandatory foundation teaching programme; this usually takes place at the acute trust and forms part of doctors' Annual Review of Competence Progression (ARCP) requirement.

Supervisors may also consider supporting foundation doctors to attend local educational activities for psychiatry trainees, such as journal clubs, case conferences or MRCPsych teaching sessions. For example, a foundation doctor could be supported to attend a teaching session on 'assessing capacity', as this is in their curriculum. Local education providers and foundation schools may also consider developing a teaching programme specifically for foundation doctors.

\section{Supervision}

Each foundation doctor will have a preassigned educational supervisor for the year and will have one clinical supervisor for each placement. Foundation doctors should aim to meet with each supervisor at the beginning and end of each rotation. In addition, clinical supervisors in psychiatry rotations are expected to deliver weekly 1 -hour face-to-face supervision. Part of the supervision process should involve a review of the foundation doctor's progress and a review of which competencies they have met from the curriculum. From the outset, clinical supervisors should agree with the foundation doctor the times of these meetings and the consultant or higher trainee who will conduct the meetings in the clinical supervisor's absence. This is particularly important in the first year of the Foundation Programme, as it helps to support doctors in the often difficult student-to-doctor transition (Steele 2013).

\section{Supervised learning events (SLES) and assessments}

Foundation doctors have to complete a number of SLEs and obtain various other assessments for a successful ARCP. SLEs are formative workplacebased assessments that involve an interaction between the trainer and the foundation doctor, leading to immediate qualitative feedback and reflective learning. They replaced workplacebased assessments (WBPAs) in the Foundation Programme from 2012. SLEs may appear superficially similar to WBPAs, but they represent a desire to make the process more formative, although this shift was not universally well understood (Rees 2014). SLEs have three components, which are outlined in Fig. 1: 
- directly observed interactions

- case-based discussions (CBDs)

- developing the clinical teacher.

The additional assessments, reports and evidence that foundation doctors must obtain for the ARCP are shown in Fig. 2. In brief, they are:

- a team assessment of behaviour (TAB)

- end-of-placement and end-of-year reports

- evidence of performance of core procedures.

All SLEs and assessments are designed to monitor foundation doctors' progress and provide a platform for further development. They are all completed in the electronic portfolio (e-portfolio), where further information about each SLE and assessment can be located. It is advised that consultant supervisors become familiar with the e-portfolio system in order to provide optimum support to their trainees. Completion of SLEs should be consultant-led where possible and should be undertaken early in the placement, when the doctor has the most to learn (Academy of Medical Royal Colleges 2016).

\section{Tasters}

Clinical exposure can be further complemented by taster days organised locally by supervisors. The Royal College of Psychiatrists suggests that posts should be developed to include five taster days in other subspecialty areas of psychiatry (Boyle 2015). These days are provided through study leave for FY2s, but the opportunity for FY1s to undertake taster days will depend on local arrangements. Clinical supervisors can maintain quality through the bespoke design of taster days, focusing on the two main objectives: namely, informing career intentions, within and outside psychiatry, and fitting with the foundation doctor's interests (UK Foundation Programme Office 2011). Examples of suitable tasters might include child and adolescent mental health services for a doctor who is interested in paediatrics as a career, or neuropsychiatry for a doctor who is interested in neurology.

In the next section we will focus on how foundation placements in psychiatry can offer doctors the opportunity to develop both generic and mentalhealth specific skills. These opportunities will also help to ensure that posts are of high quality.

\section{Supporting foundation doctors in achieving the generic and specific mental health competencies of the curriculum}

\section{MDT working}

All supervisors should aspire to give their foundation doctors a high-quality experience of

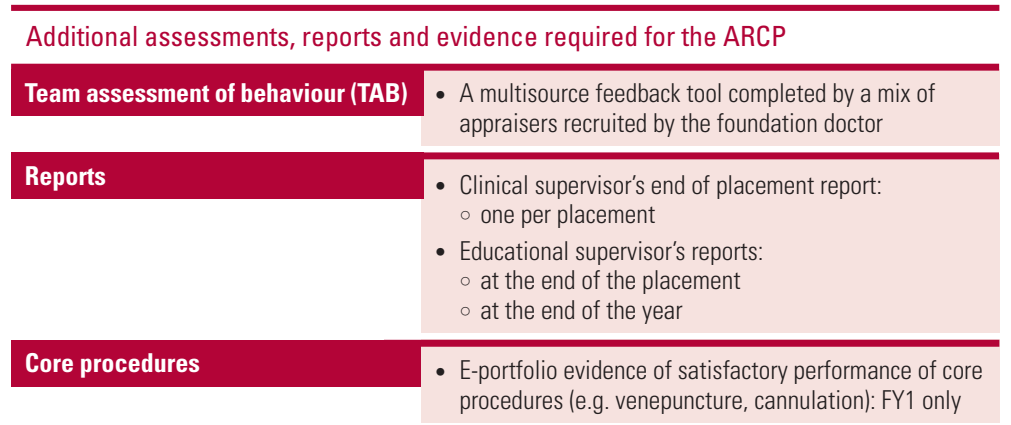

FIG 2

The additional formal assessments and evidence required for successful completion of the Annual Review of Competence Progression (ARCP) for foundation doctors (Academy of Medical Royal Colleges 2016).

MDT working. Care programme approach (CPA) meetings are a gold standard of multiprofessional working in mental healthcare, so supervisors should encourage foundation doctors to attend them. Supervisors can organise for foundation doctors to undertake joint assessments with other MDT members to give them an appreciation of the broad range of professions involved and the skills they bring to mental healthcare. Supervisors should support their foundation doctors to attend clinical team meetings and encourage them to contribute. These opportunities for MDT working should allow other team members to make reliable judgements about the foundation doctor's ability and performance. This feedback can be used in their appraisal.

\section{Communication skills}

Supervisors should develop posts that allow foundation doctors to acquire and develop communication skills. This could be done by enabling foundation doctors to work with patients, their families and other professionals in both straightforward and more complex situations. It might involve the foundation doctor explaining a treatment plan to a patient with chronic schizophrenia and an intellectual disability in a way that the patient can understand. It might involve breaking bad news, such as a new diagnosis of dementia, to a patient and their family in a sensitive and supportive manner. It is vital that foundation doctors are able to communicate empathically in this post-Francis era (Francis 2013).

\section{Reflective ability}

The Foundation Programme curriculum encourages doctors to reflect on, and learn from, both their positive and negative experiences in order to demonstrate clinical development (Academy of Medical Royal Colleges 2016). Supervisors can support the development of reflective ability 
through the 1-hour face-to-face weekly supervision. If there is the opportunity, supervisors should encourage their doctors to attend a specific Balint group for foundation doctors. Balint groups enable doctors to gain experience of reflection, listening and supporting others in the group, as well as developing a deeper understanding of their patients (Salinsky 2015).

\section{Interface with different specialties and with other professionals}

In mental health settings, the supervisor can signpost the foundation doctor to cases that will allow them to experience working across health and social care boundaries (Duncan 2015). For example, a foundation doctor could observe a core trainee assess a patient on a psychiatric ward who has a medical problem that requires discussion with the on-call medical registrar and transfer to the accident and emergency (A \& E) department. This would allow the doctor to develop an understanding of working across mental health and acute trusts. Another example might be inviting a social worker to attend a ward round for a patient about whom there are safeguarding concerns. This would allow the foundation doctor to understand more about working across mental health and social care boundaries.

\section{History-taking, mental state examination and core medical skills}

The supervisor should ensure that the foundation doctor has the opportunity to develop skills in history-taking and mental state examination through teaching, practical experience and reflection (through SLEs and supervision). Supervisors and other team members should signpost foundation doctors to opportunities to acquire core medical skills within their day-today clinical work (e.g. physical health assessments of new admissions in an in-patient setting). Local providers may like to consider developing posts that have timetabled sessions in an acute medical setting (e.g. 1 day a week on-call in a co-located acute trust).

\section{Management of patients with long-term conditions}

The Foundation Programme curriculum outlines how doctors should gain experience of managing patients with long-term conditions (Academy of Medical Royal Colleges 2016). In mental health settings, the supervisor should highlight, through teaching and supervision, the interplay between long-term physical illness, psychological factors and mental disorders. For example, the foundation doctor could undertake a case-based discussion of a patient with chronic schizophrenia and diabetes who experienced trauma in childhood.

Opportunities to have experience of holistic care in both acute presentations and in long-term conditions are likely to be widely available in all psychiatry placements.

\section{Teaching and quality improvement}

Supervisors must ensure that foundation doctors have protected time to engage in audit and quality improvement work (Cai 2009). It is recommended that half a day per week is embedded in all foundation psychiatry placement timetables to support this activity (Boyle 2015).

Supervisors and other team members should seek out opportunities for foundation doctors to teach others. For foundation doctors in psychiatry this could include teaching topics that align with their curriculum to medical students who are on psychiatry rotations, or teaching a medical topic to the MDT. It may be helpful for the foundation doctor to do this in conjunction with a senior colleague who can provide supervision and feedback. This could also be done as an SLE (developing the clinical teacher).

\section{Recognition and management of the acutely ill patient}

Foundation doctors should be supported by their supervisors to gain experience of recognising and managing patients who are acutely unwell and those who have self-harmed. Supervisors could organise for foundation doctors to conduct home visits, undertake a taster in liaison psychiatry or observe a Mental Health Act assessment. This would enable foundation doctors to gain experience of conducting risk assessments and considering underlying causes of severe mental disturbance, such as acute confusional states, psychosis and substance use/withdrawal.

\section{Medicolegal issues}

Supervisors should help their foundation doctors to acquire knowledge of medicolegal and ethical issues in healthcare. This could be, for example, through the supervisor's own teaching or through a larger programme, such as MRCPsych teaching. Teams should ensure that foundation doctors are able to gain an understanding of the Mental Capacity Act and experience of using it, for example through observing/participating in the assessment of whether a patient has capacity to consent to treatment on an in-patient ward (Nicholson 2008).

Supervisors must ensure that they provide specific teaching and supervision concerning 
the powers of detention for FY2 doctors who are on call out of hours and are deputising for the responsible clinician under the Mental Health Act.

\section{Learning opportunities}

Foundation doctors must be afforded equitable access to learning opportunities in the psychiatry placement. It is a risk that the most routine service delivery tasks such as 'scribing' in medical records during ward rounds are regularly delegated to the foundation doctor by the more senior specialty trainees. Another risk is that the foundation doctor may be forced to shadow more experienced clinicians for the duration of their placement. Teams will need to identify and support a range of experiential learning opportunities for the post to allow the foundation doctor to grow in confidence and to safely support their wider professional development as a doctor.

\section{Experience beyond the curriculum}

Some foundation doctors will wish to acquire competencies beyond the Foundation Programme over the course of their placement. They may already have decided on psychiatry as a career, or they may wish to maximise their learning opportunities. Further learning opportunities could include additional experience in psychotherapy (e.g. participation in a psychotherapy group), emergency psychiatry (e.g. observation or supervised undertaking of Section 136 assessments) and medicolegal aspects of psychiatry (e.g. observation of a Mental Health Act assessment or a tribunal).

\section{Conclusions}

The implication of the increase in the number of psychiatry posts as a result of changes to the Foundation Programme is that consultant psychiatrists and the wider team will be supervising and training these foundation doctors.

There is a need to ensure that these new psychiatry foundation posts are of high quality so that doctors have a positive experience of the specialty. Supervisors must ensure that foundation doctors are well supported by weekly clinical supervision, a well-planned induction and access to good-quality teaching programmes. Foundation doctors must be able to obtain the necessary generic and mental health-specific competencies within their respective psychiatric setting. Box 3 summarises the main foundation competencies concerned. Supervisors must provide opportunities for foundation doctors to meet patients with mental illness, particularly those with long-term conditions and those in community settings. Foundation posts should be
BOX 3 Foundation competencies that can be acquired in psychiatric settings

- Team-working within a multidisciplinary team

- Communication skills

- Reflective ability

- Interfacing with different specialties and with other professionals

- History-taking, mental state examination and core medical skills

- Management of patients with long-term conditions

- Teaching and quality improvement

- Recognition and management of the acutely ill patient

- Knowledge of medicolegal issues

developed to allow doctors to develop their teamworking and communication skills and to gain experience of navigating the boundaries of health and social care.

Throughout their careers all doctors will come into contact with patients suffering from mental illness. It is important therefore that they develop the knowledge and skills to deliver good-quality care to such patients early on in their training. The expansion of the Foundation Programme in psychiatry enables this.

\section{References}

Academy of Medical Royal Colleges (2016) The Foundation Programme Curriculum 2016. Academy of Medical Royal Colleges.

Bleakley A (2002) Pre-registration house officers and ward-based learning: a 'new apprenticeship' model. Medical Education, 36: 9-15.

Boyle A, Perry J (2015) A Guide to Psychiatry in the Foundation Programme for Supervisors. Royal College of Psychiatrists.

Cai A, Greenall J, Ding DCD (2009) UK junior doctors' experience of clinical audit in the Foundation Programme. British Journal of Medical Practitioners, 2: 42-5.

Collins J (2010) Foundation for Excellence: An Evaluation of the Foundation Programme. Medical Education England.

Davies S (2014) Annual Report of the Chief Medical Officer 2013. Public Mental Health Priorities: Investing in the Evidence. Department of Health.

Duncan E (2015) Liaison psychiatry is a good foundation for junior doctors. BMJ Careers, 9 Nov.

Foundation Programme Malta (2016) Welcome to the Foundation Programme - Malta (http://fpmalta.com). Accessed 7 November 2016.

Francis R (2013) Report of the Mid Staffordshire NHS Foundation Trust Public Inquiry: vols 1-3. TSO (The Stationery Office).

General Medical Council (2015) Foundation Programme year 1. GMC (http://www.gmc-uk.org/doctors/registration_applications/uk_ internships.asp). Accessed 7 November 2016.

Goodyear H, Bindal N, Bindal T, et al (2014) Foundation doctors' experience and views of mentoring. British Journal of Hospital Medicine, 74: $682-6$.

Health Education England (2014) Broadening the Foundation Programme: Recommendations and Implementation Guidance. February 2014. Health Education England. 


\section{$\mathrm{MCO}$ answers \\ $\begin{array}{lllll}1 b & 2 c & 3 c & 4 b & 5 d\end{array}$}

Health Education England (2015) Better Training, Better Care: Evaluation of the National Elements Report. January 2015. Health Education England.

Miles S, Kellett J, Leinster SJ (2015) Foundation doctors' induction experiences. BMC Medical Education, 15: 118

Nicholson TRJ, Cutter W, Hotopf M (2008) Assessing mental capacity: the Mental Capacity Act. BMJ, 336: 322-5.

Perry J, Boyle A, Wessely S (2016) The expansion of the psychiatry Foundation Programme. BJPsych Bulletin, 40: 223-5.

Rees CE, Cleland JA, Dennis A, et al (2014) Supervised learning events in the Foundation Programme: a UK-wide narrative interview study. BMJ Open, 4(10): e005980.

Royal College of Psychiatrists (2016) Foundation Doctor Blogs (http://www.rcpsych.ac.uk/discoverpsychiatry/foundationdoctors/ foundationdoctorblogs.aspx).

Salinsky J (2015) A Very Short Introduction to Balint Groups. Balint Society (http://balint.co.uk/about/introduction/). Accessed 7 November 2016 .
Shape of Training (2013) Securing the Future of Excellent Patient Care: Final Report of the Independent Review Led by Professor David Greenaway. Shape of Training.

Steele R, Beattie S (2013) Development of foundation year 1 psychiatry posts: implications for practice. Advances in Psychiatric Treatment, 19: 410-9.

Swanwick T, Buckley G (2014) Introduction: understanding medical education. In Understanding Medical Education: Evidence, Theory and Practice (ed T Stanwick): xv-xviii. Wiley Blackwell.

UK Foundation Programme Office (2011) Specialty Tasters in the Foundation Programme: Guidance for Foundation Schools. UK Foundation Programme Office.

UK Foundation Programme Office (2015) Rough Guide to the Foundation Programme (4th edn). UK Foundation Programme Office.

UK Foundation Programme Office (2016) UK Foundation Programme Reference Guide: May 2016. UK Foundation Programme Office (http:// www.foundationprogramme.nhs.uk/pages/home/reference-guide).

Wilson A, Feyer AM (2015) Review of Medical Intern Training: Final Report. Australian Health Ministers' Advisory Council.

\section{MCOs \\ Select the single best option for each question stem \\ 1 FY1 doctors: \\ a cannot prescribe depot medications \\ b are recommended not to prescribe in an out- patient setting \\ c can only prescribe if countersigned by a consultant \\ d can only prescribe medication for a maximum of 1 week \\ e cannot initiate new medications}

2 From 2017, the target proportion of doctors rotating through psychiatry in England by the end of their Foundation Programme years is: a $6 \%$

\section{b $22.5 \%$}

c $45 \%$

\section{d $80 \%$}

e $100 \%$.

3 The foundation competencies that all doctors would be expected to acquire in psychiatry placements do not include:

a team-working skills

b communication skills

c undertaking a seclusion review

d performing a mental state examination

e managing patients with long-term conditions.

\section{FY1 doctors:}

a can never work out of hours

b should not work on the same rotas as core trainees

c must always be supervised by a senior consultant psychiatrist on site out of hours d can undertake section 136 assessments independently when on call

e can undertake seclusion reviews independently when on call.

\section{$5 \mathrm{FY} 1$ and FY2 doctors are not required to} complete/obtain:

a mini-clinical evaluation exercises (mini-CEXs)

b core procedures

c team assessments of behaviour (TABs)

d mini-assessed clinical encounters (mini-ACEs)

e case-based discussions (CBDs). 